\title{
Efectos del disturbio antrópico sobre las poblaciones de Leprolochus birabeni (Araneae, Zodariidae) en el Chaco Seco del noroeste de Argentina
}

\author{
Víctor M. Torres ${ }^{1,2}$, Andrea X. González-Reyes ${ }^{1}$, \\ Sandra M. Rodriguez-Artigas ${ }^{1,2}$ \& José A. Corronca ${ }^{1,2}$
}

\begin{abstract}
1. Instituto para el Estudio de la Biodiversidad de Invertebrados (IEBI), Facultad de Ciencias Naturales, Universidad Nacional de Salta, Av. Bolivia 5150, Salta, CP 4400 Argentina. (torres.vicman@gmail.com)

2. Consejo Nacional de Investigaciones Científicas y Técnicas (CONICET).
\end{abstract}

\begin{abstract}
Effects of anthropogenic disturbance on the populations of Leprolochus birabeni (Araneae, Zodariidae) in the Chaco Seco northwestern Argentina. Little is known about the biology and ecology of the myrmecophagic species Leprolochus birabeni Mello-Leitão, 1942 (Zodariidae), the only representative of this genus in Argentina. This species is typical of arid environments of the north of Argentina, and their study in the Chaco Seco is relevant, because is a highly disturbed ecoregion and one of the few with dry subtropical forests in the World. The aim of this work is to study the demography of this species, considering its seasonal variation in native environments, and if the livestock grazing and the agriculture, two of the most common anthropogenic activities of the Chaco Seco ecoregion, produce changes in their populations in the province of Salta (Argentina). We consider three type of environments: native (NA); affected by cattle and goats (NP); and affected by agriculture (NC). The latter corresponds to patches of native vegetation in an agricultural matrix. Three sampling sites were selected on each type of environment, where seasonal samplings using pitfall traps were performed during autumn, winter and spring (2006) and summer (2007). We collected 549 spiders of L. birabeni, with the juveniles reaching until the $64.12 \%$ of total abundance, followed by males $(26.41 \%)$ and females $(9.47 \%)$. At landscape level, spring season showed the highest number of individuals, reaching $44.99 \%$ of the total abundance, while autumn reported the lowest percentage $(7.83 \%)$. The juveniles increase observed during the winter would indicate an adaptation to environmental conditions. The same pattern was observed in NA and NC environments, but not in NP ones. The later environments showed a decrease in the adult abundances across the seasons, while the NC populations showed an increase of male abundances during spring season with a sharp reduction of females and juveniles throughout the year. The abundance of $L$. birabeni in NA environments was higher ( $41.06 \%$ of total abundance) and the least value was reported in NC environments (24.67\%). The two most common anthropogenic activities affecting the Chaco Seco of the province of Salta modify both the seasonal changes as the representation of the different developmental stages and sexes of the native populations of $L$. birabeni, being the most damaging factor the modification of the native environments by the agriculture.
\end{abstract}

KEYWORDS. Spiders, demography, seasonal variation, population changes, Chaco ecoregion.

RESUMEN. Se conoce poco sobre la biología y la ecología de la especie mirmecófaga Leprolochus birabeni Mello-Leitão, 1942 (Zodariidae), el único representante de este género en la Argentina. Esta especie es típica de ambientes áridos del norte del país, y su estudio en el Chaco Seco es relevante, por ser una ecorregión altamente perturbada con uno de los pocos bosques subtropicales secos del mundo. El objetivo de este trabajo es estudiar la demografía de la especie teniendo en cuenta su variación estacional en ambientes nativos, y sí la ganadería y la agricultura, dos de las actividades antropogénicas más comunes de la ecorregión Chaco Seco en la provincia de Salta (Argentina) producen cambios en sus poblaciones. Se consideraron tres tipos de ambientes: nativo (NA); afectados por el ganado vacuno y caprino (NP); y afectados por la agricultura (NC). Este último correspondió a parches de vegetación nativa en medio de una matriz agrícola. En cada tipo de ambiente se consideraron tres sitios de muestreo donde se realizaron muestreos estacionales utilizando trampas de caída durante el otoño, invierno y primavera de 2006 y el verano de 2007 . Se recolectaron 549 ejemplares de $L$. birabeni, siendo la mayoría juveniles con el $64,12 \%$ de la abundancia total, seguidos por machos $(26,41 \%)$ y hembras $(9,47 \%)$. A nivel de paisaje, la primavera presentó el mayor número de individuos, llegando al 44,99\% de la abundancia total; mientras que el otoño mostró el porcentaje más bajo $(7,83 \%)$. El incremento de los juveniles durante el invierno podría indicar una adaptación de los estadios de desarrollo a las condiciones ambientales. El mismo patrón se observó en ambientes NA y NC, pero no en los NP. Estos últimos mostraron una disminución en la abundancia de adultos a lo largo del año; mientras que las poblaciones $\mathrm{NC}$ mostraron un incremento en la abundancia de los machos durante la primavera, con una fuerte reducción de las hembras y los juveniles durante todo el año. La abundancia de L. birabeni en ambientes NA fue mayor (41,06\% de la abundancia total), siendo los valores mínimos registrados en NC (24,67\%). Las dos actividades antropogénicas más comunes que afectan al Chaco Seco de la provincia de Salta modifican tanto los cambios estacionales como la representación de las diferentes etapas de desarrollo y sexos de las poblaciones nativas de $L$. birabeni, siendo la modificación de los ambientes nativos por la agricultura, el factor más perjudicial.

PALABRAS CLAVE. Arañas, demografía, variación estacional, cambios poblacionales, ecorregión Chaco. 
El uso antropogénico del suelo es uno de los factores más importantes en el cambio global (SALA et al., 2000), produciendo pérdida de especies debido a la modificación del paisaje (TsCHARNTKE et al., 2002; FAHRIG, 2003; FISCHER \& LindenMAYER, 2007). La fragmentación actúa como uno de los factores clave de los cambios que ocurren en la estructura comunitaria de los organismos y en sus dinámicas poblacionales (FAHrig \& GREZ, 1996; LAURANCE \& BIERREGAARD, 1997). Ésta involucra la reducción de hábitats, un incremento en el número de parches y la separación entre ellos (aislamiento), y un decremento en el tamaño de los hábitats remanentes (FAHRIG, 2003). Esta pérdida irreversible de la diversidad biológica por las actividades antropogénicas puede tener consecuencias impredecibles para el equilibrio del ambiente, ya que muchos servicios ecosistémicos están declinando de manera acelerada (Purvis \& Hector, 2000). Por ello, la rápida tasa de extinción de las especies es uno de los principales problemas ambientales asociado a las actividades humanas.

Varios autores han mostrado que la fragmentación genera cambios en el tamaño poblacional, la riqueza de especies y la distribución de los individuos (BOLGER et al., 2000; VentiCINQUe \& Fowler, 2001; Mestre \& GASNIER, 2008; GonzÁlez et al., 2011; STEFANI \& DEL-Claro, 2014). De esa manera, un grupo taxonómico como el de las arañas, que está fuerte y predeciblemente influenciado por el tipo de hábitat y los patrones en el uso del suelo (UETZ, 1975; WeEks \& Holtzer, 2000; Grill et al., 2005; JANSEN et al., 2013), es relevante para evaluar estos cambios ambientales. La fragmentación produce cambios en la composición de la comunidad de plantas y una variación en la disponibilidad de presas, influenciando la diversidad de las arañas (WISE, 1993; GibB \& Hochuli, 2002). Por ello, han tenido una amplia aceptación en los estudios ecológicos como indicadores de calidad ambiental (Clausen, 1986; ChurChill, 1998; Pinkus-Rendón et al., 2006; HsieH \& Linsenmair, 2011).

Por otra parte, los factores bióticos (competencia, depredadores, calidad y cantidad de recursos) y abióticos (temperatura, precipitación, entre otros) son importantes en los sistemas naturales ya que pueden influir sobre la dinámica de sus poblaciones (DENNo et al., 2002; GRATTON \& DENNO, 2003; Romero \& VASCONCELlos-Neto, 2003). De ellos, la estacionalidad es un factor clave que actúa sobre la riqueza y la abundancia de las comunidades de arañas (WoLDA, 1988; RODRIGUES et al., 2015), ejerciendo un efecto sobre la tasa de crecimiento o el tamaño de las poblaciones (GASNIER et al., 2002).

El Chaco es un bosque seco neotropical que se encuentra entre los biomas más amenazados del mundo (GRAU et al., 2008). Debido a su condición de vulnerable (Dinerstein et al., 1995; BertonatTi \& CoRCuera, 2000), el Chaco argentino es la principal ecorregión del país por la importancia de su conservación; aunque es el principal escenario de la actual expansión de la frontera agropecuaria, que avanza sobre sus ambientes naturales (TORRELLA \& AdÁmoli, 2005; PET, 2010; Morello et al., 2012). Como consecuencia, su diversidad se encuentra entre las más amenazadas del país debido principalmente a la reducción y la fragmentación del hábitat (TORRELLA \& ADÁMOLI, 2005; PIQUER-Rodríguez et al., 2015).

Leprolochus birabeni Mello-Leitão, 1942 es una especie mirmecófaga de zodárido que se distribuye desde el norte de Brasil hasta la región central de Argentina (JOCQUÉ, 1988). Es la única especie del género presente en el país (World Spider Catalog, 2016), siendo muy común en el Chaco y el Monte de Argentina (JoCQué, 1988; Flores et al., 2004; Pompozzi et al., 2011). Se conoce poco sobre la biología y la ecología de los zodáridos en general y más aún de sus especies en particular. Leprolochus birabeni muestra interés porque es típica de estas zonas áridas que están sufriendo cambios ambientales producto de las actividades antrópicas. Además, es una especie que ha mostrado ser muy abundante e indicadora de la calidad ambiental para la ecorregión del Chaco en el noroeste de Argentina (I. G. Cruz, com. pers.). Así, cualquier cambio en la abundancia de sus poblaciones puede brindar información acerca de la calidad ambiental en esta ecorregión. Es por eso, que el objetivo de este trabajo es abordar el estudio demográfico de la especie, considerando la variación estacional de sus poblaciones, analizando los cambios que estas sufren por el efecto de la ganadería y la agricultura en el Chaco Seco de la provincia de Salta, Argentina. Así, predecimos que hay un patrón estacional en la abundancia de L. birabeni en el Chaco Seco, mostrando una mayor representatividad numérica en las estaciones más cálidas; y que los distintos disturbios antrópicos que se ejercen sobre los ambientes nativos del Chaco Seco salteño producen una reducción de la abundancia de la especie.

\section{MATERIALES Y MÉTODOS}

Área de estudio. Correspondió a un sector del Chaco Seco de la porción central de la provincia de Salta (Argentina) comprendida en el Valle del Río Juramento, a margen derecha del río, desde el dique Cabra Corral (Departamento La Viña, $25^{\circ} 18^{\prime} 18.52$ 'S; 6517’30.04”O) hasta $75 \mathrm{~km}$ al noreste sobre la Ruta Provincial secundaria ${ }^{\circ} 8$ (Departamento General Güemes, 2439'14”S; 6559'40’'W) (Fig. 1). El clima de la región es subtropical cálido, con una gran amplitud térmica diaria asociada a la variación estacional incluyendo inviernos con frentes fríos que originan heladas y una precipitación anual que varía entre 500 y $700 \mathrm{~mm}$. La variación en la temperatura y precipitación durante el período de estudio (Fig. 2), muestra que el invierno registró las temperaturas más bajas y la primavera los valores más altos, mientras que el verano alcanzo el valor más alto de precipitación (142 mm). Estos datos provienen de la estación meteorológica INTA, departamento Cerrillos (Salta, Argentina), ubicado a $40 \mathrm{~km}$ al oeste de la zona de estudio, no siendo posible registrar datos de estos parámetros para cada sitio muestreado. La vegetación dominante está representada por Schinopsis haenkeana Engl. (horco-quebracho), especies de cactus como Trichocereus terscheckii (Parm. ex Pfeiff.) Britton \& Rose, y Stetsonia coryne (Salm-Dyck) Britton \& Rose, y la asterácea Carduus 


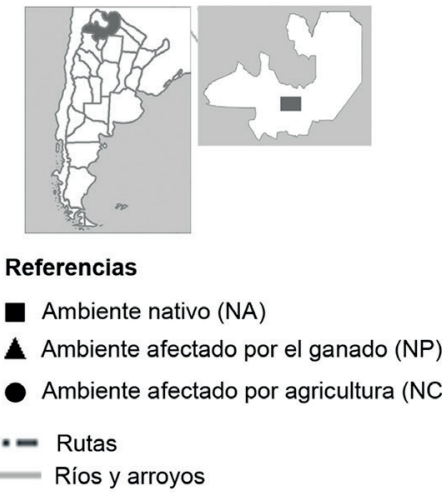

Ríos y arroyos
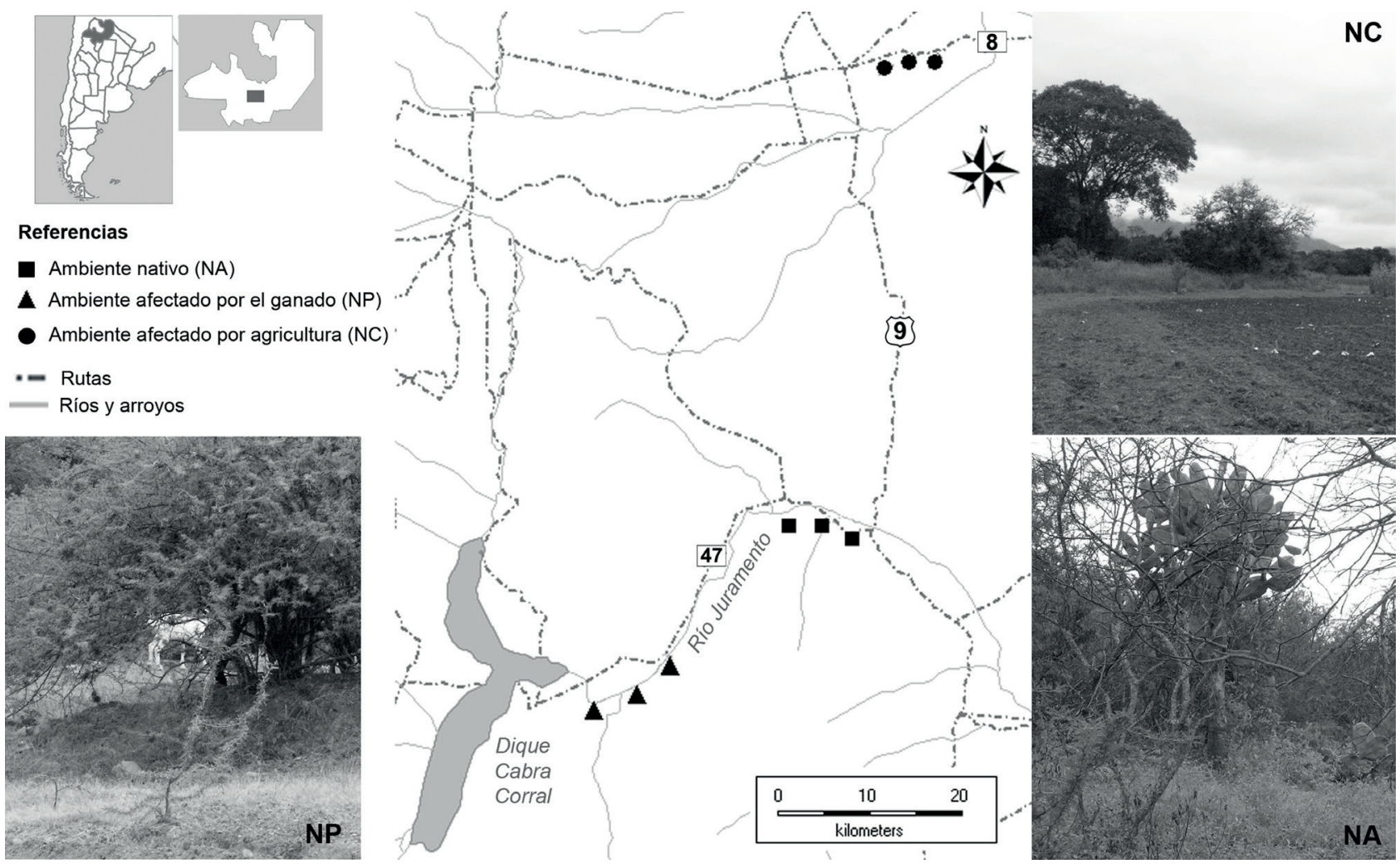

Fig. 1. Mapa de la zona de estudio con los sitios de muestreo de los ambientes nativo (NA), afectado por el ganado (NP) y afectado por agricultura (NC) del Chaco Seco de la provincia de Salta, Argentina.

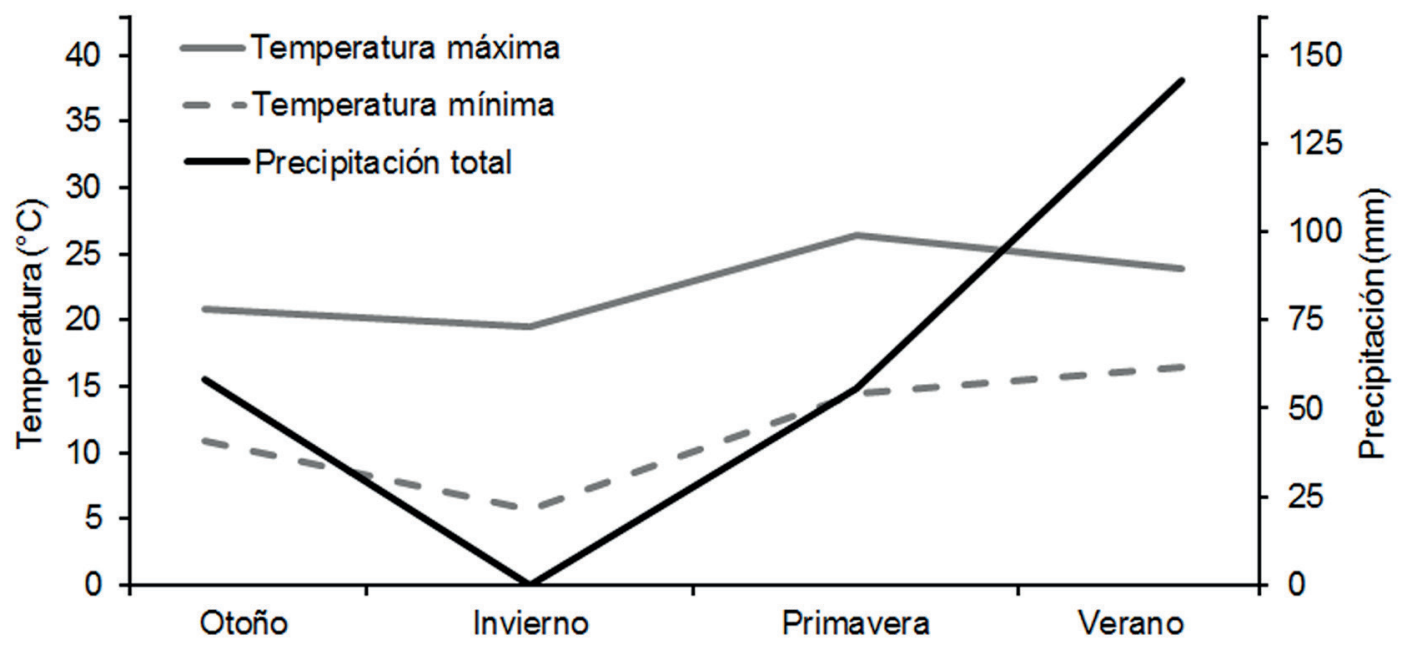

Fig. 2. Variación de la temperatura y precipitación durante el período de estudio en el Chaco Seco de la provincia de Salta, Argentina [Los datos provienen de la estación meteorológica INTA (Cerrillos, Salta), los valores de temperatura corresponden a promedios mensuales y valores de precipitación al total registrado en los meses muestreados; Abril (otoño), Agosto (invierno), Noviembre (primavera) del 2006, y Marzo (verano) del 2007].

nutans L., también se encuentra Aspidosperma quebrachoblanco Schltdl. (quebracho blanco), Ziziphus mistol Griseb. (mistol) y arbustos de los géneros Acacia sp. y Capparis sp. (Morello et al., 2012). En los ambientes pastoreados abundan especies exóticas como Tithonia tubaeformis (Jacq.) Cass. (pasto cubano), que llega a más de tres metros de altura (Chebez, 2005); como así también Acacia visco Griseb. (arca), Tipuana tipu (Benth.) Kuntze (tipa) y Enterolobium contortisiliquum (Vell.) Morong (pacará), importantes en los pastizales sobrepastoreados, en las parcelas de cultivos abandonadas y en los bosques ribereños. A ellos, se les suma en los ambientes degradados, Opuntia quimilo K. Schum. (quimil) (Morello et al., 2012). Los sitios pastoreados por ganado muestreados en este estudio, mostraron pastizales degradados que fueron colonizados por vinales (Prosopis ruscifolia Griseb.) y duraznillos (Ruprechtia triflora Griseb.), mostrando un estrato arbustivo denso con cactáceas arbóreas. Muestreo. El muestreo incluyó tres tipos de ambientes 
del Chaco Seco salteño: ambiente nativo (NA), sitios con vegetación nativa sin alteración antrópica; ambiente afectado por el ganado (NP), correspondiente a sitios con ganado vacuno y caprino o rastros de ellos (pastoreo, pisoteo, huellas y heces); y ambiente afectado por la agricultura (NC), correspondientes a pequeños parches nativos del Chaco Seco en medio de un paisaje circundante netamente agrícola representado principalmente por cultivos de soja, poroto y maíz. La separación entre ambientes fue de $10 \mathrm{~km}$ y en cada uno de ellos se seleccionaron tres sitios de muestreo geo-referenciados y distanciados entre sí por al menos 3,5 $\mathrm{km}$. En ellos se realizaron muestreos estacionales: otoño, invierno y primavera (2006), y verano (2007). En cada sitio se colocaron 10 trampas de caída separadas entre sí por $10 \mathrm{~m}$ a lo largo de una transecta lineal, y con actividad de 7 días por estación durante la misma semana en los tres ambientes. Las trampas fueron recipientes plásticos de $7.5 \times 12.2 \times 5.2 \mathrm{~cm}$ (diámetro superior $\times$ profundidad $\times$ diámetro inferior), con solución salina $[\mathrm{ClNa}(\mathrm{kg})$ - agua (lt) en una proporción 1:8, más gotas de detergente]. El material recolectado, rotulado, fue colocado en bolsas de polietileno con etanol al 70\%. Los ejemplares fueron registrados y separados según el sitio, estación, estado del desarrollo (juvenil/adulto) y sexo. Los especímenes recolectados fueron depositados en la colección IEBI-MCN (Instituto para el Estudio de la Biodiversidad de Invertebrados-Museo de Ciencias Naturales, Universidad Nacional de Salta, Argentina).

Análisis de datos. La abundancia promedio por trampa de L. birabeni fue analizada de dos maneras diferentes. Se comparó la abundancia estacional de todo el muestreo y por tipo de ambiente por estado/sexo, y también se comparó la abundancia de juveniles, machos y hembras entre ambientes por cada estación y en todo el muestreo en el Chaco Seco salteño. Estas comparaciones se llevaron cabo por medio del Análisis de la Varianza Multivariado (MANOVA, Wilks' lambda) con el programa PAST ver.2.14 (HAMMER et al., 2003). Los valores de abundancia fueron transformados a Ln $(x+1)$ para cumplir con el supuesto de normalidad. Para aquellos valores significativos de Wilks' lambda, se continuó con comparaciones pareadas por medio del test de Hotelling, los valores de probabilidad se ajustaron por Bonferroni por medio del programa PAST ver.2.14 (HAMMER et al., 2003).

\section{RESULTADOS}

Se recolectaron 549 ejemplares de L. birabeni, los juveniles representaron el $64.12 \%$ de los individuos registrados; mientras que los machos alcanzaron el $26.41 \%$ de la abundancia total. Durante el muestreo se pudo observar que el máximo incremento de la abundancia de la especie se registró durante la primavera, alcanzando valores del $44.99 \%$ de la abundancia total, y el menor porcentaje de ejemplares fue reportado durante el otoño mostrando valores del $7.83 \%$. El MANOVA reportó que existen diferencias de significación estadística en la abundancia de la especie entre estaciones (Tab. I, ver Paisaje) en el Chaco Seco salteño, lo que fue corroborado para los ambientes nativos y para los parches nativos rodeados por un paisaje agrícola, no así para los ambientes afectados por el pastoreo del ganado (Tab. I, Total NA, NC y NP). Los resultados muestran que, en los ambientes NA y NC, la primavera y el verano seguidas por el invierno resultaron ser las estaciones con mayor número de individuos (test Hotelling, $\mathrm{p}<0.05$ ). En esta última estación, la abundancia estuvo representada principalmente por los juveniles, especialmente en NA. En cambio, en el ambiente NP la ganadería afectó directamente al patrón de la abundancia de la especie debido a que no se observó diferencias de significación estadística entre las abundancias promedio por trampa a lo largo de las estaciones muestreadas (Tab. I). Además, los juveniles de L. birabeni resultaron más abundantes en todas las estaciones con respecto a los adultos (Tab. I), excepto en el invierno y la primavera para NC (Fig. 3). Por otra parte, los machos fueron más abundantes que las hembras en todas las estaciones, mostrando un marcado incremento durante la primavera (Tab. I, test Hotelling, $\mathrm{p}<0.05)$.

Tab. I. Comparación mediante MANOVA, de la abundancia promedio ( \pm error estándar) por trampa (total y estado/sexo) de Leprolochus birabeni MelloLeitão, 1942 entre estaciones del año a nivel de paisaje y por ambiente muestreado en el Chaco Seco de la provincia de Salta, Argentina (NA, ambiente nativo; NP, ambiente afectado por el ganado; NC, ambiente afectado por agricultura; en negrita se indican los valores de $p$ significativos).

\begin{tabular}{|c|c|c|c|c|c|c|c|c|}
\hline & Otoño & Invierno & Primavera & Verano & Wilks' lambda & GL & $\mathrm{F}$ & $p$ \\
\hline Total NA & $0.30 \pm 0.11$ & $1.77 \pm 0.38$ & $3.13 \pm 0.76$ & $2.30 \pm 0.55$ & 0,5702 & 9 & 2,392 & 0,0184 \\
\hline Machos & $0.03 \pm 0.03$ & $0.10 \pm 0.06$ & $1.23 \pm 0.37$ & $0.40 \pm 0.13$ & 0,4254 & 9 & 3,876 & 0,0004 \\
\hline Hembras & $0.10 \pm 0.07$ & $0.10 \pm 0.06$ & $0.17 \pm 0.07$ & $0.36 \pm 0.18$ & 0,8079 & 9 & 0,844 & 0,5783 \\
\hline Juveniles & $0.17 \pm 0.07$ & $1.57 \pm 0.34$ & $1.73 \pm 0.49$ & $1.53 \pm 0.42$ & 0,64 & 9 & 1,851 & 0,0710 \\
\hline Total NP & $0.63 \pm 0.21$ & $1.26 \pm 0.26$ & $2.06 \pm 0.63$ & $1.87 \pm 0.49$ & 0,6432 & 9 & 1,831 & 0,2195 \\
\hline Machos & $0.10 \pm 0.06$ & $0.10 \pm 0.56$ & $0.37 \pm 0.16$ & $0.33 \pm 0.16$ & 0,7608 & 9 & 1,095 & 0,3755 \\
\hline Juveniles & $0.50 \pm 0.18$ & $1.03 \pm 0.22$ & $1.50 \pm 0.50$ & $1.37 \pm 0.35$ & 0,6877 & 9 & 1,532 & 0,1505 \\
\hline Total NC & $0.50 \pm 0.16$ & $0.57 \pm 0.20$ & $3.03 \pm 0.99$ & $0.87 \pm 0.40$ & 0,5897 & 9 & 2,232 & 0,02762 \\
\hline Machos & $0.17 \pm 0.07$ & $0.27 \pm 0.14$ & $1.53 \pm 0.63$ & $0.20 \pm 0.12$ & 0,7149 & 9 & 1,362 & 0,2187 \\
\hline Hembras & $0.03 \pm 0.03$ & $0.03 \pm 0.03$ & $0.40 \pm 0.16$ & 0 & 0,6256 & 9 & 1,958 & 0,0548 \\
\hline Juveniles & $0.30 \pm 0.12$ & $0.27 \pm 0.14$ & $1.10 \pm 0.32$ & $0.66 \pm 0.36$ & 0,6633 & 9 & 1,692 & 0,1038 \\
\hline Paisaje & $0.56 \pm 0.11$ & $1.50 \pm 0.20$ & $3.24 \pm 0.60$ & $2.19 \pm 0.35$ & 0,3471 & 9 & 5,017 & $<0.0001$ \\
\hline Machos & $0.10 \pm 0.03$ & $0.16 \pm 0.05$ & $1.04 \pm 0.25$ & $0.31 \pm 0.13$ & 0,4079 & 9 & 4,104 & 0,0002 \\
\hline Juveniles & $0.32 \pm 0.07$ & $0.96 \pm 0.15$ & $1.44 \pm 0.26$ & $1.19 \pm 0.22$ & 0,4348 & 9 & 3,759 & 0,0005 \\
\hline
\end{tabular}


El patrón de abundancia de L. birabeni en los ambientes nativos del Chaco Seco salteño mostró que la abundancia de la especie fue mayor en la primavera y baja en el otoño (Fig. 3 , Tab. I, Test Hotelling $\mathrm{p}<0.05$ ). Las hembras fueron más abundantes en el verano y los machos durante la primavera, mientras que los juveniles sólo disminuyeron su número en el otoño (Fig. 3); aunque únicamente se observó diferencias de significación estadística en los cambios estacionales de las abundancias de los machos (Tab. I). Los sitios afectados por el ganado y el paisaje agrícola mostraron cambios en el patrón observado en los ambientes nativos estudiados. Así, los ambientes $\mathrm{NC}$ propiciaron un incremento de la abundancia
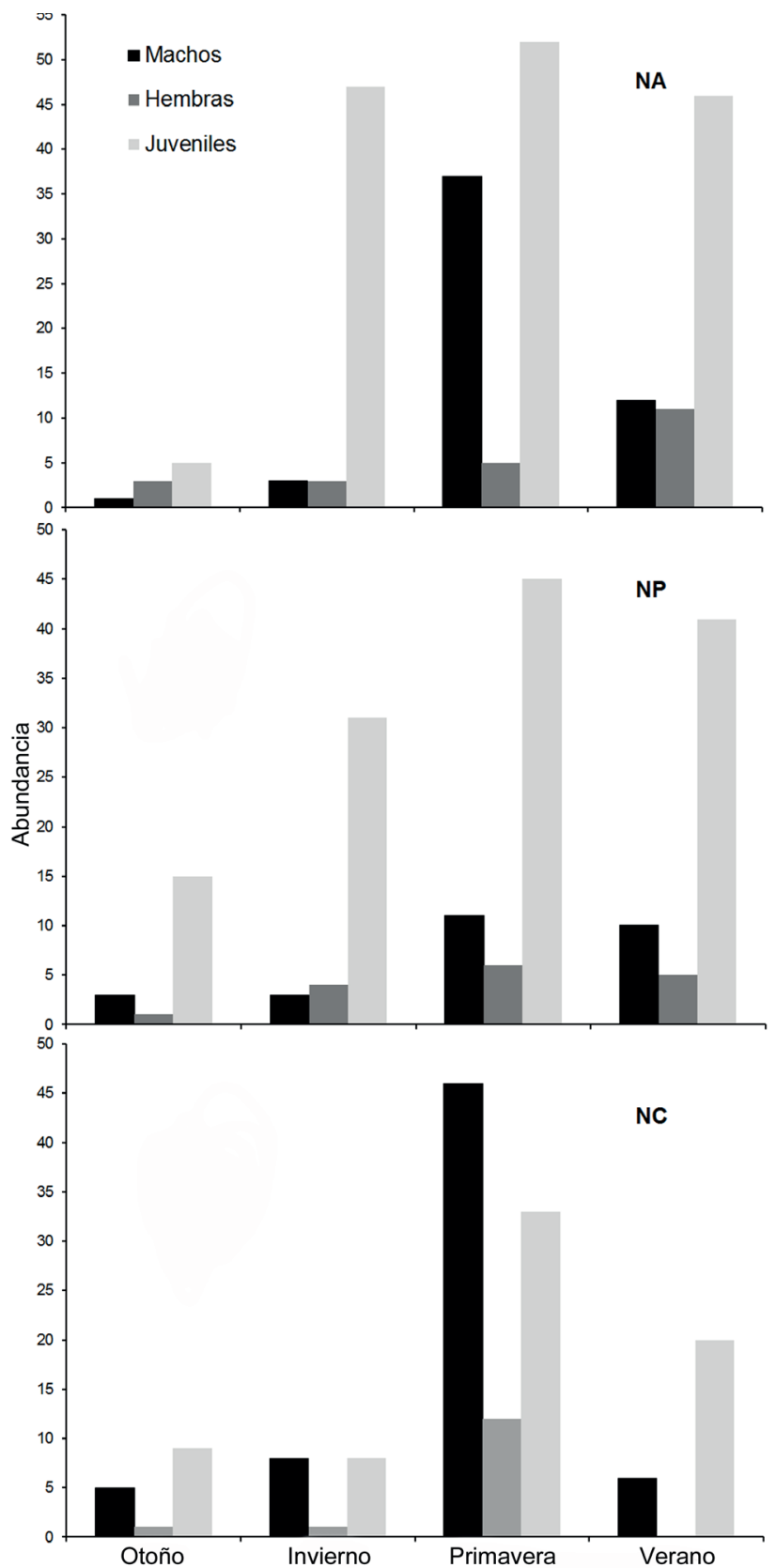

Fig. 3. Abundancia de machos, hembras y juveniles de Leprolochus birabeni Mello-Leitão, 1942 por estación en cada ambiente muestreado en el Chaco Seco de la provincia de Salta, Argentina. (NA, ambiente nativo; NP, ambiente afectado por el ganado; $\mathrm{NC}$, ambiente afectado por agricultura). de los machos (Fig. 4), particularmente durante la primavera (Fig. 3), y una reducción de las hembras a lo largo del año, en particular en el verano donde no se registraron individuos de este sexo (Fig. 3, Tab. I). En cambio, en la misma figura, se puede observar que los ambientes NP mostraron cambios en el patrón de abundancia de los adultos de L. birabeni, más que de los juveniles, con respecto a lo registrado en NA.

Las actividades antrópicas no sólo produjeron cambios en el patrón de variación de la abundancia de los estados/ sexos de las poblaciones nativas del Chaco Seco salteño, sino también en la abundancia total registrada. De esa manera, los parches nativos rodeados por un paisaje agrícola redujeron en un tercio, y los sitios NP en un $22 \%$, la abundancia total reportada en los ambientes nativos no alterados. Estos datos fueron corroborados por el Test de Hotelling $(p<0.05)$, que mostró diferencias de significación estadística entre las abundancias de los ambientes muestreados. Además, al comparar los distintos estados/sexos de L. birabeni entre los tres ambientes considerados se pudo observar cambios en la abundancia de los juveniles durante el invierno; y de los machos, hembras y juveniles durante la primavera mostrando diferencias de significación estadística entre ellos (Tab. II, Fig. 3). Por lo tanto las actividades antropogénicas estarían influyendo sobre las abundancias de las poblaciones en estaciones contrastantes climáticamente donde se registran los mayores valores de abundancia para la especie, produciendo una variación en el patrón general observado para sus poblaciones nativas.

\section{DISCUSIÓN}

Nuestros resultados sugieren que las actividades antrópicas desarrolladas en el Chaco Seco del Valle de Lerma, Salta (Argentina) modifican los cambios estacionales de las poblaciones nativas de $L$. birabeni, una especie indicadora (I. G. Cruz, com. pers.) de la calidad ambiental de esta ecorregión en el noroeste argentino. Así, los principales cambios no sólo

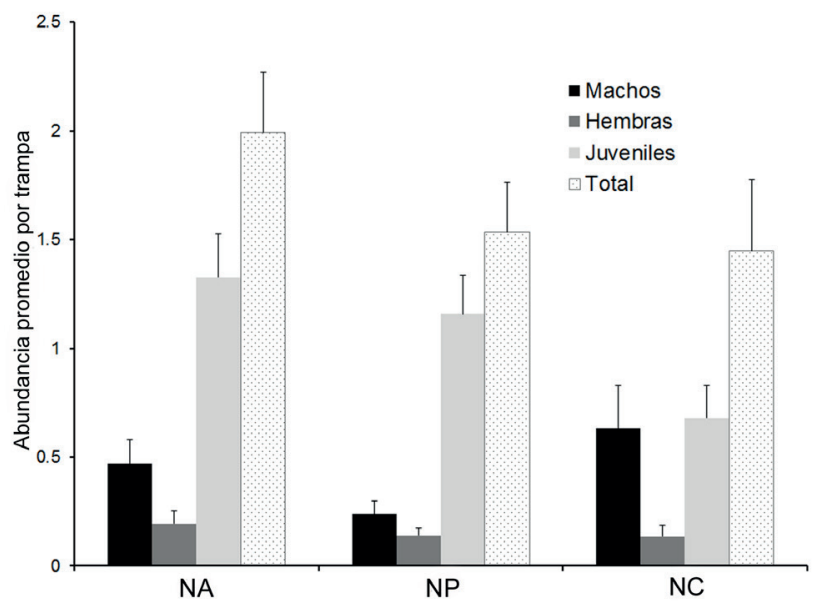

Fig. 4. Abundancia promedio ( \pm error estándar) por trampa de machos, hembras, juveniles, y total de Leprolochus birabeni Mello-Leitão, 1942 en los ambientes del Chaco Seco de la provincia de Salta, Argentina. (NA, ambiente nativo; NP, ambiente afectado por el ganado; NC, ambiente afectado por agricultura). 
Tab. II. Comparación mediante MANOVA, de la abundancia promedio ( \pm error estándar) por trampa (total y estado/sexo) de Leprolochus birabeni Mello-Leitão, 1942 entre los ambientes estudiados, por estación del año muestreada y total en el Chaco Seco de la provincia de Salta, Argentina. (En negrita se indican los valores de $p$ significativos, los valores de abundancia promedio por trampa se visualizan en Tabla I).

\begin{tabular}{ccccc}
\hline & Wilks' lambda & GL & F & $p$ \\
\hline Otoño & & & & \\
Machos & 0,7871 & 6 & 1,059 & 0,3992 \\
Hembras & 0,9203 & 6 & 0,3535 & 0,9045 \\
Juveniles & 0,683 & 6 & 1,750 & 0,1288 \\
Invierno & & & & \\
Machos & 0,7431 & 6 & 1,334 & 0,2598 \\
Hembras & 0,8436 & 6 & 0,740 & 0,6201 \\
Juveniles & 0,2343 & 6 & 8,884 & $<\mathbf{0 . 0 0 0 1}$ \\
Primavera & & & & \\
Machos & 0,5593 & 6 & 2,809 & $\mathbf{0 , 0 1 9 6}$ \\
Hembras & 0,5719 & 6 & 2,686 & $\mathbf{0 , 0 2 4 4}$ \\
Juveniles & 0,5785 & 6 & 2,623 & $\mathbf{0 , 0 2 7 3}$ \\
Verano & & & & \\
Machos & 0,6409 & 6 & 2,076 & 0,0727 \\
Hembras & 0,6893 & 6 & 1,704 & 0,1395 \\
Juveniles & 0,6893 & 6 & 1,704 & 0,1395 \\
Total & & & & \\
Machos & 0,8571 & 6 & 2,939 & $\mathbf{0 , 0 0 8 9}$ \\
Hembras & 0,8908 & 6 & 2,181 & $\mathbf{0 , 0 5 0 0}$ \\
Juveniles & 0,7993 & 6 & 4,347 & $\mathbf{0 , 0 0 0 4}$ \\
Total & 0,75663 & 6 & 5,494 & $<\mathbf{0 . 0 0 0 1}$ \\
\hline
\end{tabular}

se registran en una reducción en la abundancia de la especie en los ambientes antropizados, ya que estas actividades (ganadería, agricultura, urbanización, entre otras) influyen sobre las abundancias relativas de las especies (CHAPIN III et al., 2000), sino también sobre la representatividad de los diferentes estados del desarrollo y sexos.

Las poblaciones nativas de L. birabeni en el Chaco Seco salteño muestran un patrón estacional donde si bien los juveniles son abundantes a lo largo del año, su número se reduce de manera marcada en el otoño, coincidiendo también con un decremento de la abundancia de los adultos, en particular de los machos. El repentino incremento de los juveniles de esta especie en el invierno, puede atribuirse a un periodo de hibernación de los juveniles o los huevos, situación que ha sido observada para otras familias de arañas (JIMÉNEZ \& NAVARRETE, 2010; WelCh et al., 2011). El incremento de los machos en los períodos cálidos y húmedos (primavera y verano), como así también de los juveniles, mostraría que ellos tienen su período reproductivo en esos momentos del año. Durante la época reproductiva los machos están probablemente más activos en la búsqueda de pareja, por lo que la probabilidad de ser capturados por las trampas de caída es alta (ADIs, 2002; Mineo et al., 2010), como quedó registrado en nuestros resultados. La misma explicación podría aplicarse a los juveniles, teniendo en cuenta que ellos son realmente abundantes en esos momentos del año y están a la búsqueda de diferentes recursos (hábitat, refugios, presas, entre otros) de manera activa, como así también es el período de dispersión y colonización de nuevos ambientes. Así, en los estudios de arañas es común reportar una alta proporción de juveniles (SAmu et al., 1997; BRIERTON et al., 2003), pudiendo llegar a valores del $80 \%$ del total de ejemplares recolectados. Los cambios de abundancia observados en las poblaciones de L. birabeni para el Chaco Seco salteño, en particular el incremento de la misma en los períodos de primavera-verano, también han sido reportados por FLORES et al. (2004) en una área natural protegida de la provincia de Mendoza (Argentina); para poblaciones de una especie no identificada de Leprolochus en la sabana brasileña (MINEO et al., 2010); para dos especies del género Zodarion Walckenaer, 1826 (Zodariidae) para Europa (PeKÁr et al., 2005); y para especies de Lycosidae y Miturgidae en Argentina, Uruguay y Brasil (González et al., 2014; Stefani \& Del-Claro, 2014; Pompozzi et al., 2014).

Las variables climáticas reinantes en el Chaco Seco salteño, con precipitación de $500-700 \mathrm{~mm}$ anuales y con una gran amplitud térmica diaria asociada a la variación estacional (Morello et al., 2012), pueden influir en la estacionalidad de la flora y de la fauna. Se conoce que la precipitación y la temperatura pueden ser los factores más importantes que conducen los cambios en las abundancias y en la riqueza de especies de artrópodos en áreas subtropicales y tropicales (Wolda, 1988; BASSET, 1991; PinheIro et al., 2002; Mineo et al., 2010; Pompozzi et al., 2014; Rodrigues et al., 2015). Por otro lado, la vegetación de la ecorregión representada típicamente por un bosque xerófilo estacional abierto, donde concurren especies perennifolias y caducifolias, también podría influir sobre los cambios estacionales de las arañas, por su marcada dependencia al hábitat. Estos factores pueden actuar sobre sus poblaciones y en su selección de hábitats, y posiblemente también están jugando un papel importante en la variación estacional de las poblaciones de L. birabeni en el área de estudio. Además, el incremento de la vegetación en los períodos cálidos y húmedos puede asociarse con una mayor disponibilidad de presas en esta época del año (UETZ, 1976; RieCHERT \& LuCZAK, 1982), que podría explicar también la variación estacional observada para la especie en los ambientes nativos estudiados.

La ganadería se extendió en los ambientes del Chaco no ocupados por la agricultura (ADÁMOLI et al., 2004), modificando no sólo la comunidad vegetal de la zona sino también el paisaje. Esta actividad es de antigua data en el valle de Lerma de la provincia de Salta. Ella produce efectos sobre la estructura de la vegetación, actuando de manera directa sobre la disponibilidad de presas e indirectamente sobre las condiciones microclimáticas a nivel del suelo (GIBSON et al., 1992; Bell et al., 2001; DenNis et al., 2001; PASChETTA et al., 2013). A pesar de ello, esta actividad reduce la abundancia total de L. birabeni en menor proporción que el paisaje agrícola, pero tiene un mayor efecto sobre la abundancia de adultos. Por otro lado, los resultados muestran que esta actividad reduce la variación estacional de las poblaciones de L. birabeni en el Chaco Seco de la provincia de Salta.

En el caso de los parches nativos del Chaco Seco inmersos en una matriz agrícola, el cambio en la abundancia fue mayor que en los ambientes pastoreados por el ganado. En aquellos, se puede observar una disminución en el número de hembras a lo largo del año, excepto en la primavera, que es la época reproductiva. Esto es coincidente con un marcado 
incremento de los machos, que pudieron ser registrados en las trampas por su marcada actividad para ir a la búsqueda de su pareja reproductiva. Durante el verano, época en la cual el porcentaje de hembras es máximo en los ambientes nativos del Chaco Seco, ellas no fueron registradas en los parches de bosque nativo en medio del paisaje agrícola. Ese patrón de ausencia y/o reducción en la abundancia de L. birabeni en estos ambientes podría deberse a un efecto de posible sensibilidad a los insecticidas que se aplican en los campos cultivados y su deriva a los ambientes nativos circundantes (Prieto-Benítez \& MÉndez, 2011). Este efecto ha sido reportado en ambientes circundantes a cultivos de soja en Argentina por LiLJESTHROM et al. (2002) y Beltramo et al. (2006). Por otro lado, el cambio del paisaje nativo por disturbios de origen antrópicos tiene un efecto negativo sobre la propia heterogeneidad del hábitat. Ello se traduce en cambios en la riqueza y abundancias de las arañas, principalmente cuando ellos son transformados en agroecosistemas (Prieto-Benítez \& MÉndez, 2011). Así, la presencia de parches nativos pequeños y muy distantes entre sí que quedan en el paisaje agrícola estudiado, cada vez más creciente, podrían afectar de manera marcada a las poblaciones de L. birabeni a lo largo del tiempo. Este efecto de reducción de la abundancia de los depredadores, y entre ellos las arañas, ha sido reportado por GoNZÁLEZ et al. (2011) en fragmentos de pequeño tamaño del Chaco Seco de la provincia de Córdoba (Argentina). Este efecto es aún mayor cuando el grupo tiene una dieta especializada como L. birabeni. KотіAно et al. (2005) han sugerido que la especialización en la dieta es uno de los factores relacionados a la extinción de las especies en los paisajes modificados.

Concluyendo, en este trabajo se amplía el conocimiento sobre el patrón fenológico y demográfico de L. birabeni, única especie del género sudamericano (JocQué, 1988) presente en Argentina. Además, se muestran los cambios estacionales que sufre la abundancia de la especie en ambientes nativos y en aquellos afectados por actividades relacionadas con el uso del suelo en el Chaco Seco del noroeste de Argentina. Estos datos, sumados a nuevos que puedan surgir en futuros estudios, serán de gran utilidad para seguir profundizando sobre el papel que esta especie cumple como indicadora del estado de conservación de los ambientes de esta ecorregión en el norte de Argentina.

Agradecimientos. Queremos expresar nuestro agradecimiento al CIUNSa (Consejo de Investigación de la Universidad Nacional de Salta) por su apoyo financiero, al CONICET (Consejo Nacional de Investigaciones Científicas y Técnicas) por su constante apoyo y a la Secretaría de Medio Ambiente del Gobierno de la Provincia de Salta por permitirnos realizar la recolección de material entomológico en el área en estudio, y a S. Cravero por brindarnos los datos de temperatura y precipitación del periodo de estudio.

\section{REFERENCIAS BIBLIOGRAFICAS}

AdÁmoli, J.; Torrella, S. \& GinZburg, R. 2004. Diagnóstico Ambiental del Chaco Argentino. Gestión Integrada y Desarrollo Sostenible para reducir la degradación social, económica y ambiental del Gran Chaco Americano. Argentina. Buenos Aires, Secretaría de Ambiente y Desarrollo Sustentable de la Nación Argentina. 105p.
ADIS, J. 2002. Recomended sampling techniques. In: ADIs, J. ed. Amazonian Arachnida and Myriapoda. Sofia, Pentsoft Publishers, p. 555-576.

BASSET, Y. 1991. The seasonality of arboreal arthropods foraging within an Australian rainforest tree. Ecological Entomology 16(3):265-278.

Bell, J. R.; Wheater, C. P. \& Cullen, W. R. 2001. The implications of grassland and heathland management for the conservation of spider communities: a review. Journal of Zoology 255:377-387.

Beltramo, J.; Bertolaccini, I. \& González, A. 2006. Spiders of soybean crops in Santa Fé province, Argentina: Influence of surrounding spontaneous vegetation on lot colonization. Brazilian Journal of Biology 66:891-898.

Bertonatti, C. \& Corcuera, J. 2000. Situación Ambiental Argentina 2000. Buenos Aires, Fundación Vida Silvestre. 436p.

Bolger, D. T.; Suarez, A. V.; Crooks, K. R.; Morrison, S. A. \& Case, T. J. 2000. Arthropods in urban habitat fragments in Southern California: area, age, and edge effects. Ecological Applications 10(4):1230-1248.

Brierton, B. M.; Allen, M. D. C. \& Jennings, D. T. 2003. Spider fauna of sugar maple and white ash in northern and central New York State. Journal of Arachnology 31(3):350-362.

Chapin III, F. S.; Zavaleta, E. S.; Eviner, V. T.; NAYlor, R. L.; VitouseK, P. M.; Reynolds, H. L.; Hooper, D. U.; Lavorel, S.; Sala, O. E.; HobBIE, S. E.; MACK, M. C. \& DíAZ, S. 2000. Consequences of changing biodiversity. Nature 405(6783):234-242.

Chebez, J. C. 2005. Guía de las reservas naturales de la Argentina. Noroeste. Buenos Aires, Albatros. 255p.

Churchill, T. B. 1998. Spiders as ecological indicators in the Australian tropics: family distribution patterns along rainfall and grazing gradients. In: SELDEN, P. A. ed. Proceedings of the 17th European Colloquium of Arachnology, Edinburgh 1977. Burnham Beeches, Bucks, British Arachnological Society, p. 325-330.

Clausen, I. H. 1986. The use of spiders (Araneae) as ecological indicators. Bulletin of the British Arachnological Society 7:83-86.

Dennis, P.; Young, M. R. \& BentLey, C. 2001. The effects of varied grazing management on epigeal spiders, harvestment and pseudoscorpions of Nardus stricta grassland in upland Scotland. Agriculture, Ecosystems and Environment 86:39-57.

Denno, R. F.; Gratton, C.; Peterson, M. A.; Langellotto, G. A.; Finke, D. L. \& HUBERTY, A. F. 2002. Bottom-up forces mediate natural-enemy impact in a phytophagous insect community. Ecology 83:1443-1458.

Dinerstein, E.; Olson, D.; Graham, D.; Webster, A.; Primm, S.; BooKbinder, M. \& LeDEC, G. 1995. Una evaluación del estado de conservación de las ecorregiones terrestres de América Latina y el Caribe. Washington DC, IUCN and World Bank. 133p.

FAHRIG, L. 2003. Effects of habitat fragmentation on biodiversity. Annual Review of Ecology, Evolution and Systematics 34:487-515.

FAHRIG, L. \& GREZ, A. A. 1996. Population spatial structure, human-caused landscape changes and species survival. Revista Chilena de Historia Natural 69:5-13.

FisCheR, J. \& LindenMAYeR, D. B. 2007. Landscape modification and habitat fragmentation: a synthesis. Global Ecology and Biogeography 16(3):265-280.

Flores, G. E.; LaGos, S. J. \& Roig-JuÑent, S. 2004. Artrópodos epigeos que viven bajo la copa del algarrobo (Prosopis flexuosa) en la reserva Telteca (Mendoza, Argentina). Multequina 13:71-90.

Gasnier, T. R.; Torres-SAnchez, M. P.; Azevedo, C. S. \& HöFer, H. 2002. Adult size of eight hunting spider species in central Amazonia: temporal variations and sexual dimorphisms. Journal of Arachnology 30:146-154.

GibB, H. \& Hochuli, D. F. 2002. Habitat fragmentation in an urban environment: large and small fragments support different arthropod assemblages. Biological Conservation 106:9-100.

Gibson, C. W. D.; Hambler, C. \& Brown, V. K. 1992. Changes in spider (Araneae) assemblages in relation to succession and grazing management. Journal of Applied Ecology 29:132-142.

González, E.; SAlvo, A. \& Valladares, G. R. 2011. Artrópodos fitófagos y entomófagos asociados a la vegetación responden diferencialmente a la fragmentación del Chaco Seco. Revista de la Asociación Argentina de Ecología de Paisajes 2(2):48-55.

González, M.; Costa, F. G. \& Peretti, A. V. 2014. Strong phenological differences between two populations of a Neotropical funnel-web wolf spider. Journal of Natural History 48(35-36):2183-2197. 
Gratton, C. \& Denno, R. F. 2003. Seasonal shift from bottom-up to top-down impact in phytophagous insect populations. Oecologia 134:487-495.

Grau, R. H.; Gasparri, N. I. \& Aide, M. 2008. Balancing food production and nature conservation in the Neotropical dry forests of northern Argentina. Global Change Biology 14(5):985-997.

Grill, A.; Knoflach, B.; Cleary, D. F. R. \& Kati, V. 2005. Butterfly, spider, and plant communities in different land-use types in Sardinia, Italy. Biodiversity and Conservation 14(5):1281-1300.

Hammer, O.; Harper, D. A. T. \& Ryan, P. D. 2003. PAST: Paleontological Statistics. Version 1.18. Available at $<$ http://folk.uio.no/ohammer/past $>$.

HsieH, Y. L. \& LinsenMaIR, K. E. 2011. Underestimated spider diversity in a temperate beech forest. Biodiversity and Conservation 20(13):29532965.

Jansen, R.; Makaka, L.; Little, I. T. \& DippenaAR-Schoeman, A. 2013. Response of ground-dwelling spider assemblages (Arachnida, Araneae) to Montane Grassland management practices in South Africa. Insect Conservation and Diversity 6(5):572-589.

Jiménez, M. L. \& Navarrete, J. G. 2010. Fauna de arañas del suelo de una comunidad árida-tropical en Baja California Sur, México. Revista Mexicana de Biodiversidad 81(2):417-426.

JoCQUÉ, R. 1988. An updating of the genus Leprolochus (Araneae: Zodariidae). Studies on Neotropical Fauna and Environment 23(2):77-87.

Kotiaho, J. S.; Kaitala, V.; Komonen, A. \& Päıvinen, J. 2005. Predicting the risk of extinction from shared ecological characteristics. Proceedings of the National Academy of Sciences of the United States of America 102(6): 1963-1967.

LAurance, W. F. \& BierregaARD, R. O. 1997. Tropical forest remnants: ecology, management, and conservation of fragmented communities. Chicago, University of Chicago Press. 632p.

LiljesthröM, G.; Minervino, E.; Castro, D. \& GonzÁlez, A. 2002. La comunidad de arañas del cultivo de soja en la provincia de Buenos Aires, Argentina. Neotropical Entomology 3(2):197-210.

Mestre, L. A. M. \& Gasnier, T. R. 2008. Populações de aranhas errantes do gênero Ctenus em fragmentos florestais na Amazônia Central. Acta Amazonica 38:159-164

Mineo, M. F.; Del-Claro, K. \& Brescovit, A. D. 2010. Seasonal variation of ground spiders in a Brazilian Savanna. Zoologia 27(3):353-362.

Morello, J.; Matteucci, S. D.; Rodriguez, A. F. \& Silva, M. E. 2012. Ecorregiones y Complejos Ecosistémicos Argentinos. Buenos Aires, Orientación gráfica editora. 753p.

Paschetta, M.; La Morgia, V.; Masante, D.; Negro, M.; Rolando, A. \& IsAIA, M. 2013. Grazing history influences biodiversity: a case study on ground-dwelling arachnids (Arachnida: Araneae, Opiliones) in the Natural Park of Alpi Marittime (NW Italy). Journal of Insect Conservation 17(2):339-356.

PekÁr, S.; Král, J.; Malten, A. \& Komposch, C. 2005. Comparison of natural histories and karyotypes of two closely related ant-eating spiders, Zodarion hamatum and Z. italicum (Araneae, Zodariidae). Journal of Natural History 39(19):1583-1596

Pinheiro, F.; Diniz, I. R.; Coelho, D. \& Bandeira, M. P. S. 2002. Seasonal pattern of insect abundance in the Brazilian cerrado. Austral Ecology 27(2):132-136

Pinkus-Rendón, M. A.; León-Cortés, J. L. \& IbArRA-Nuñez, G. 2006. Spider diversity in a tropical habitat gradient in Chiapas, Mexico. Diversity and Distribution 12(1):61-69.

Piquer-Rodríguez, M.; Torella, S.; Gavier-Pizarro, G.; Volante, J.; Somma, D.; Ginzburg, R. \& Kuemmerle, T. 2015. Effects of past and future land conversions on forest connectivity in the Argentine Chaco. Landscape Ecology 30(5):817-833.

Pet - Plan Estratégico Territorial Argentina. 2010. Provincia de Salta. Available at $<$ http://www.chienhwa.net/PET/SP/Salta.pdf $>$.

Pompozzi, G.; Copperi, S.; Schwerdt, L. \& Ferretti, N. 2014. On the ecology of the cursorial spider Odo bruchi (Araneae: Zoridae) in a grassland natural reserve from central Argentina. Revista de Biología Tropical 62(1):111-117.
Pompozzi, G. A.; Tizón, F. R. \& Pelaéz, D. V. 2011. Effects of different frequencies of fire on an epigeal spider community in Southern Caldenal, Argentina. Zoological Studies 50(6):718-724.

Prieto-Benítez, S. \& MÉndeZ, M. 2011. Effects of land management on the abundance and richness of spiders (Araneae): A meta-analysis. Biological Conservation 144(2):683-691.

Purvis, A. \& Hector, A. 2000. Getting the measure of biodiversity. Nature 405(6783):212-219.

RIECHERT, S. E. \& LUCZAK, J. 1982. Spider foraging: behavioral responses to prey. In: Witt, P. N. \& Rovner, J. S. eds. Spider comunication. Mechanisms and ecological significance. New Jersey, Princenton University Press, p. 353-385.

Rodrigues, E. N. L.; Mendonça JR., M. de S.; Rodrigues, P. E. S. \& OtT, R. 2015. Diversity, composition and phenology of araneid orb-weavers (Araneae, Araneidae) associated with riparian forests in southern Brazil. Iheringia, Série Zoologia 105(1):53-61.

Romero, G. Q. \& VAsconcellos-Neto, J. 2003. Natural history of Misumenops argenteus (Thomisidae): seasonality and diet on Trichogoniopsis adenantha (Asteraceae). Journal of Arachnology 31:297-304.

Sala, O. E.; Chapin, F. S.; Armesto, J. J.; Berlow Bloomflied, J.; Dirzo, R.; Hubersandwald, E.; Huenneke, L. F.; JaCKson, R. B.; Kinzig, A.; Leemans, R.; Lodge, D. M.; Mooney, H. A.; Oesterheld, M.; Poff, N. L.; Sykes, M. T.; Walker, B. H.; Walker, M. \& Wall, D. H. 2000. Global biodiversity scenarios for the year 2100. Science 287:1770-1774.

SAMU, F.; RÁCZ, V.; ERdélyi, C. \& BALÁzs, K. 1997. Spiders of the foliage and herbaceous layer of an IPM apple orchard in Kecskemét-Szarkás, Hungary. Biological Agriculture and Horticulture 15(1-4):131-140.

Stefani, V. \& Del-Claro, K. 2014. The effects of forest fragmentation on the population ecology and natural history of a funnel-web spider. Journal of Natural History 49(3-4):211-231.

Torrella, S. A. \& AdÁmoli, J. 2005. Situación Ambiental de la Ecorregión del Chaco Seco. In: Brown. A.; Martínez Ortiz, U.; Acerbi, M. \& Corcuera, J. eds. La Situación Ambiental Argentina 2005. Buenos Aires, Fundación Vida Silvestre Argentina, p. 75-82.

Tscharntke, T.; Steffan-Dewenter, I.; Kruess, A. \& Thies, C. 2002. Contribution of small habitat fragments to conservation of insect communities of grassland-cropland landscapes. Ecological Applications 12(2):354-363.

Uetz, G. W. 1975. Temporal and spatial variation in species diversity of wandering spiders (Araneae) in deciduous forest litter. Environmental Entomology 4(5):719-724.

UETZ, G. W. 1976. Gradient analysis of spider communities in a streamside forest. Oecologia 22(4):373-385.

Venticinque, E. M. \& Fowler, H. G. 2001. Local extinction risks and asynchronies: the evidence for a metapopulation dynamics of a social spider, Anelosimus eximius (Araneae, Theridiidae). In: BIERrEGAARD JR, R. O.; Gascon, C.; Lovejoy, T. E. \& Mesquita. R. eds. Lessons from Amazonia: the ecology and conservation of a fragmented forest. New Haven, Yale University Press, p. 187-198.

Weeks, R. D. \& Holtzer, T. O. 2000. Habitat and season in structuring ground-dwelling spider (Araneae) communities in a shortgrass steppe ecosystem. Environmental Entomology 29(6):1164-1172.

Welch, K. D.; Crain, P. R. \& Harwood, J. D. 2011. Phenological dynamics of web-building spider populations in alfalfa: implications for biological control. The Journal of Arachnology 39:244-249.

Wise, D. H. 1993. Spiders in Ecological Webs. Cambridge, Cambridge University Press. 328p.

Wolda, H. 1988. Insect seasonality: Why? Annual Review of Ecology and Systematic 19:1-18.

World SpIDer CATAlOG. 2016. World Spider Catalog. Natural History Museum Bern. Available at $<$ http://wsc.nmbe.ch $>$. Accessed on 07 March 2016. 